\title{
Pathogenicities of Rice Blast (Pyricularia oryzae Cavara) Isolates From Kenya
}

\author{
Yoshimichi Fukuta, ${ }^{1, \dagger}$ Mary Jeanie Telebanco-Yanoria, ${ }^{2}$ Nagao Hayashi, ${ }^{3}$ Seiji Yanagihara, ${ }^{4}$ Catherine Wanjiku Machungo, ${ }^{5}$ \\ and Daigo Makihara ${ }^{6}$ \\ ${ }^{1}$ Tropical Agricultural Research Front, Japan International Research Center for Agricultural Sciences, Maezato, Ishigaki, \\ Okinawa 907-0002, Japan \\ ${ }^{2}$ International Rice Research Institute, Los Baños Laguna, The Philippines \\ ${ }^{3}$ National Institute of Agrobiological Sciences, Tsukuba, Ibaraki 305-8602, Japan \\ ${ }^{4}$ Japan International Research Center for Agricultural Sciences, Tsukuba 305-8686, Japan \\ ${ }^{5}$ Ahero Irrigation Research Station, National Irrigation Board, Ahero, Kisumu, Kenya \\ ${ }^{6}$ International Center for Research and Education in Agriculture, Nagoya University, Chikusa, Nagoya 464-8601, Japan
}

\begin{abstract}
A total of 99 isolates of rice blast (Pyricularia oryzae Cavara) were collected from 2010 to 2015 from four regions in Kenya: Kirinyaga County and Embu County, Kisumu County, Tana River County, and Mombasa County. The pathogenicities of these isolates were clarified based on the reaction patterns of Lijiangxintuanheigu and differential varieties (DVs) targeting 23 resistance genes. The frequency of virulent isolates was high for DVs for Pib, Pia, Pii, Pi3, Pi5(t), Pik-s, Pik-m, Pil, Pik-h, Pik, Pik-p, Pi7(t), Pi19(t), and Pi2O(t); low for DVs for Pish, Pi9(t), $P i z-5$, and Piz-t; and intermediate for the remaining DVs for Pit, Piz,

Pita-2, Pita, and Pi12(t). These blast isolates were classified into three cluster groups: Ia, Ib, and II. The frequencies of virulent isolates to DVs for Pit, Pii, Pik-m, Pil, Pik-h, Pik, Pik-p, Pi7(t), Piz, and Pi12(t) differed markedly between clusters I and II, and those of DVs for Pib, Pit, Pia, Pi3, Pita-2, Pita, and Pi2O(t) differed between Ia and Ib. The frequencies of cluster groups in the four geographical regions were different. A total of 62 races were found, with 19 blast isolates categorized into one race (U63-i7-k177-z00-ta003), whereas the other races included only some isolates in each.
\end{abstract}

Rice blast, caused by the pathogen Pyricularia oryzae Cavara, is one of the most serious diseases affecting rice worldwide (Zeigler et al. 1994). The use of resistant cultivars is the most economical method to control this disease in rice (Oryza sativa $\mathrm{L}$.). However, using such cultivars has a limited effect as a result of the breakdown of resistance genes, with more virulent blast races occurring. The interaction between host resistance and blast fungus virulence can be explained by the gene-for-gene theory: every resistance gene in the host corresponds to an avirulence gene in the pathogen (Flor 1971; Silué et al. 1992). Based on this theory, rice differential varieties (DVs) have been developed to distinguish races of the blast fungus

${ }^{\dagger}$ Corresponding author: Y. Fukuta; zen@affrc.go.jp

Y. Fukuta and M. J. Telebanco-Yanoria contributed equally to this work.

Funding: This study was conducted under six research projects: "Development of Rice Production Technologies in Africa" from 2011 to 2015, "Rice Innovation for Environmentally Sustainable Production Systems" from 2011 to 2015, and "Development of Sustainable Technologies to Increase Agricultural Productivity and Improve Food Security in Africa" from 2016 to 2020 in the Japan International Research Center for Agricultural Sciences; the Global Rice Science Partnership Research Project "Methodologies and New Resources for Genotyping and Phenotyping of African Rice Species and Their Pathogens for Developing Strategic Disease Resistance Breeding Programs" (MENERGEP) from 2012 to 2014; and the Bilateral Joint Research Project between Japan and Kenya projects "Interdisciplinary Study on the Rice Blast Outbreak in Mwea Irrigation Scheme in Kenya" from 2011 to 2013 and "Development of Rice Blast Disease Control Technology Through Characterization of Rice Blast Fungus and Rice Cultivars in Kenya" from 2013 to 2015 supported by the Japan Society for the Promotion of Science and the National Commission For Science, Technology, and Innovation.

*The $\boldsymbol{e}$-Xtra logo stands for "electronic extra" and indicates that one supplementary figure and two supplementary tables are published online.

The author(s) declare no conflict of interest.

Accepted for publication 30 June 2019.

(C) 2019 The American Phytopathological Society by their different patterns of reaction to different strains of pathogen. DVs can be used to determine the blast pathogen population structure and identify new blast races.

There are four rice cultivation regions in Kenya: Kirinyaga County and Embu County, Kisumu County, Tana River County, and Mombasa County. Widespread irrigation systems have been constructed in Kirinyaga County, Embu County, Kisumu County, and in the delta area of Tana River County, whereas small irrigated or rainfed paddy fields comprise the rice-growing areas of Mombasa County. Rice productivity in Kenya is low and this has been attributed to various factors, including biotic and abiotic stresses (Kouko 1997; Onyango 2014). With respect to biotic stresses, rice blast disease is the major constraint to rice production in Kenya because of its wide geographic distribution and destructiveness under unfavorable environmental conditions (Kouko 1997; Ministry of Agriculture 2014). Kihoro et al. (2013) surveyed rice blast in Mwea (Kirinyaga County) and found the disease to be widely distributed in that region.

Kariaga et al. (2016) collected blast fungus from Mwea (Kirinyaga County); Homabay, Yala Swamp, Ahero, and West Kano (Kisumu County); Bunyalla, Mumias, and Namble (Busia County); and Msambweni, Kaloleni, and Tana River Delta (Tana River Country) in Kenya. They tried to characterize the regional differences in fungal cultures, such as conidia and conidiophores, and they estimated the distributions of different blast populations in these regions based on the observations of infections of eight cultivars. However, the pathogenicities of these blast isolates were not clarified. Nyongesa et al. (2016) reported that DVs comprising monogenic lines with a Lijiangxintuanheigu (LTH) background (Tsunematsu et al. 2000) and near-isogenic lines (NILs) with a CO 39 genetic background (Telebanco-Yanoria et al. 2010) for resistance genes Pib, Pit, Pish, Pi5(t), Pik-s, Pik-m, Pil, Pik-h, Pik, Pik-p, Piz, Piz-5, Piz-t, $P i 12(\mathrm{t})$, and Pita-2 were relatively resistant to the populations of blast fungus at Mwea (Kirinyaga County), West Kano (Kisumu County), and Gamba (Tana River County), under natural infection. The number of DVs infected by the blast fungus varied among these three regions, and different races/populations were estimated to be distributed in these regions. The study by Nyongesa et al. (2016) was the first report to detail pathogenicities of the blast fungus in Kenya. However, those surveys were limited to only observations 
of natural infections of the DVs, and pathological evaluations of blast fungus and the differentiations of races were not clarified.

In previous pathogenicity studies, nine DVs (including Shin 2 for an unknown resistance gene, Aichiasahi for Pia, Ishikarishiroge for $P i i$, Kanto 51 for $P i k$, Tsuyuake for $P i k-m$, Fukunishki for $P i z$, Yahiromochi for Pita, Pi No. 4 for Pita-2, and Toride 1 for Piz-t) were used by Yamada et al. (1976, 1979), and 12 DVs (including Shin 2, BL1 for Pib, Aichiasahi, K59 for Pit, Fujisaka 5 for Pii, Kusabue for Pik, K60 for Pik-p, Tsuyuake, Fukunishki, Toride 1, K1 for Pita, and Pi No. 4) were used by Kiyosawa (1984) in Japan. This Japanese DV set for 12 resistance genes (Pia, Pib, Pit, Pii, Pik-s, Pik, Pik-m, Pik-p, Piz, Pita, Pita-2, and Piz-t) (Kiyosawa 1981, 1984) was used for pathogenicity tests in Vietnam (Noda et al. 1999). The CO 39 NILs for Pil, Pil-LAC(t), Pil-TTP(t), Piz-5, Pi3, Pi4a,Pi4b(t)(= Pita), Pi4a-PKT(t), and Pi4-TTP(t) and LTH NILs for Pib, Pik-m, Pik-p, Pik, Pita, and Pita-2 were used in Thailand (Mekwatanakarn et al. 2000). Twelve DVs for Pish, Pia, Pib, Pit, Pii, Pi3, Pi5(t), Pik-s, Pik, Pik-h, Pik-m, Pik-p, Pil, Piz, Piz-5, Piz-t, and Pi2O(t) were used in the Philippines (Telebanco-Yanoria et al. 2008). The LTH NILs for Pib, Pik-m, Pik-p, Pik, Pita, and Pita-2 and CO 39 NILs for Pi3, Pi13, Piz-5, and Pita were used in China (Chen et al. 2001). However, several Japanese DVs were found to harbor additional resistance gene(s) to the target genes in their genetic backgrounds. Hayashi et al. (1998) identified Pi19(t) in Shin 2, BL1, Aichiasahi, K59, Fujisaka 5, Kusabue, K60, Tsuyuake, Fukunishiki, Toride 1, and K1. Kato et al. (2004) also reported that Shin 2 harbors Pish and Pik-s; K59 and Fujisaka 5 have Pik-s; and Fukunishiki, Toride 1, and BL1 have Pish as well. CO 39 has Pia in the genetic background (Tsunematsu et al. 2000). Thus, the Japanese DVs established by Yamada et al. (1976, 1979) and Kiyosawa $(1981,1984)$ and the CO 39 NILs have limitations in identifying the pathogenicity of blast isolates because of the reactions of additional resistance gene(s) in the genetic backgrounds.

Tsunematsu et al. (2000) developed the monogenic lines as a new set of DVs targeting 23 resistance genes: Pish, Pib, Pit, Pia, Pii, Pi3, Pi5(t), Pik-s, Pik-m, Pil, Pik-h, Pik, Pik-p, Pi7(t), Pi9(t), Piz, Piz-5, Piz-t, Pita-2, Pita, Pi12(t), Pi19(t), and Pi2O(t). These monogenic lines were used to introduce single resistance genes into the genetic background of a Chinese Japonica rice cultivar, LTH, to reduce the effect of the genetic background. As advanced DVs, Telebanco-Yanoria et al. (2010) bred NILs of LTH targeting 11 resistance genes: Pib, Piz-5, Pi9(t), Pi3, Pia, Pik-s, Pik, Pik-h, Pi7(t), Pita, and Pita-2. These monogenic lines and LTH NILs covered 23 resistance genes and are the most effective materials for use as international DVs.

Hayashi and Fukuta (2009) proposed a new international system using monogenic lines for designating races of the rice blast pathogen. Races are designated by the reaction patterns of LTH (susceptible control) and 25 monogenic lines targeting 23 resistance genes. This set of international DVs and a new designation system has been used to evaluate the pathogenicities of blast isolates and to clarify the differentiations of blast races in Western Africa (Odjo et al. 2014), northeastern China (Wang et al. 2013), Cambodia (Fukuta et al. 2014), the United States (Wang et al. 2015), Japan (KawasakiTanaka et al. 2016), and Bangladesh (Khan et al. 2016). Using the set of common DVs and the designation system of blast races allows information on blast races from different countries or regions to be compared, and we can understand the global differentiation of blast races and can characterize the blast population in each area.

In the present study, the pathogenicities of blast isolates collected from different regions in Kenya were tested using the set of international DVs developed by Tsunematsu et al. (2000) and TelebancoYanoria et al. (2010), and races were named following the designation system developed by Hayashi and Fukuta (2009). The differences in blast races among four rice-growing regions of Kenya are discussed.

\section{Materials and Methods}

Blast sampling and single spore isolation. A total of 99 isolates of $P$. oryzae were collected from the infected leaves ( 95 isolates) and panicles (four isolates) of rice (O. sativa L.) cultivars grown in irrigated lowland and upland fields at harvest seasons, or the nursery at seeding stage, in four regions of Kenya from 2010 to 2015:
Kirinyaga County and Embu County, Kisumu County, Tana River County, and Mombasa County (Supplementary Table S1).

Single spores isolated from these samples were incubated on moist filter paper in a Petri dish at room temperature for $24 \mathrm{~h}$ following the protocol of Hayashi et al. (2009). Colonies from single conidia were grown on water agar for 5 to 7 days, and two to three cut pieces of each single colony were transferred onto sterile filter paper on rice straw agar medium. The fungi were grown on the filter paper and then dried and stored on the filter paper at $-20^{\circ} \mathrm{C}$.

Inoculation. To produce inocula, stock isolates (paper discs) were cultured on oatmeal agar medium. The inoculated plates were incubated at 25 to $28^{\circ} \mathrm{C}$ for 12 to 14 days. The culture was scraped with a sterilized toothbrush, and the plates were exposed to continuous light for 4 to 5 days to induce heavy sporulation. Conidia were dislodged from the incubated plates into sterilized distilled water containing $0.01 \%$ Tween 20 by gently rubbing with a paint brush. Spore suspensions were filtered through four layers of gauze mesh and the concentration was adjusted to $1 \times 10^{5}$ conidia/ml using a hemacytometer (Hayashi et al. 1998).

Inoculation was performed following the methods of Bonman et al. (1986) and Hayashi et al. (2009). Seedling cell trays (cells $16 \mathrm{~mm}$ in diameter $\times 25 \mathrm{~mm}$ deep, in a $5 \times 7$ array) containing 20- to 21-dayold seedlings (approximately four- to five-leaf stage) of three of the 25 DVs and LTH were placed on a rotating plate, and the seedlings were sprayed uniformly with $10 \mathrm{ml}$ of spore suspension per tray using an electric atomizer (0.3-mm spray nozzle) at $0.1 \mathrm{MPa}$. Inoculated seedlings were incubated in a dew chamber at $25^{\circ} \mathrm{C}$ for $20 \mathrm{~h}$ and then transferred to a greenhouse maintained at a temperature of $25 \pm 1^{\circ} \mathrm{C}$ and relative humidity of 70 to $80 \%$. The process of inoculation and evaluation was performed twice using three plants each.

Scoring of infection and race designation. The degree of infection of each DV and LTH in each of the blast isolates was evaluated at 7 days after inoculation by assigning a score of 0 to 5 , where 0 indicates no evidence of infection; 1 indicates brown specks smaller than $0.5 \mathrm{~mm}$ in diameter, with no sporulation; 2 indicates brown specks about 0.5 to $1 \mathrm{~mm}$ in diameter, with no sporulation; 3 indicates roundish to elliptical lesions about 1 to $3 \mathrm{~mm}$ in diameter with a gray center surrounded by brown margins, with lesions capable of sporulation; 4 indicates typical spindle-shaped blast lesions capable of sporulation, $3 \mathrm{~mm}$ or longer with necrotic gray centers and watersoaked or reddish brown margins, with little or no coalescence of lesions; and 5 indicates lesions as in 4 but with about half of one or two leaf blades killed by the coalescence of lesions. The most infected leaf was investigated among three plants, and then the mean of two inoculations was used as the representative data of infection in each DV or susceptible control. The plant reactions were further categorized by the method of Hayashi and Fukuta (2009) as resistant (in most cases, infection scores were 0 to 2 ) or as susceptible (in most cases, scores were 3 to 5). The only DV categorized as susceptible with scores of 2 to 5 was IRBL5-M for the resistance gene Pi5(t), and the only DVs categorized as susceptible with scores of 4 to 5 were IRBLsh-B for Pish and IRBLta2-Pi for Pita-2.

Designation of blast isolates was determined following the international designation system using the reaction patterns of the $25 \mathrm{DVs}$ and LTH. These DVs and LTH were divided into five groups as follows: U, i, k, z, and ta. Group U includes LTH and four DVs (IRBLaA for Pia, IRBLsh-B for Pish, IRBLb-B for Pib, and IRBLt-K59 for Pit). Group i includes three DVs [IRBLi-F5 for Pii, IRBL3-CP4 for $P i 3$, and IRBL5-M for Pi5(t)] with the Pii locus on chromosome 9. Group $\mathrm{k}$ includes seven DVs [IRBLks-F5 for Pik-s, IRBLk-Ka [LT] for $P i k$, IRBLkm-Ts for Pik-m, IRBLkp-K60 for Pik-p, IRBLkh-K3[LT] for $P i k$ - $h$, IRBL1-CL for $P i 1$, and IRBL7-M for $P i 7(\mathrm{t})]$ with the $P i k$ region on chromosome 11. Group z includes four DVs [IRBLz-Fu for Piz, IRBLz5-CA for Piz-5, IRBLzt-T for Piz- $t$, and IRBL9-W for Pi9(t)] with the Piz region on chromosome 6 . Group ta includes seven DVs [IRBLta-Kl for Pita, IRBLta-CPl for Pita, IRBLta2-Pi for Pita-2, IRBLta2-Re for Pita-2, IRBL12-M for Pi12(t), IRBL19-A for Pi19(t), and IRBL20-IR24 for Pi2O(t)] with the Pita region on chromosome 12. The 21 resistance genes in groups i, k, z, and ta are multiple alleles or are located in the same 
chromosomal regions. The other four genes (Pia on chromosome 11, Pish and Pit on chromosome 1, and Pib on chromosome 2) are located in different chromosomal regions and are gathered with LTH in group U. In this study, the monogenic lines IRBLkh-K3 and IRBLk-Ka used by Hayashi and Fukuta (2009) were replaced with two LTH NILs, IRBLkh-K3[LT] and IRBLk-Ka[LT], respectively.

Blast races were designated by using Gilmour's method (Gilmour 1973). The DVs in each of the five groups U, i, k, z, and ta were divided into one to three subunits, with one to three DVs (genes) in each subunit (see Table 1). To each of the DVs within each subunit, we applied codes 1,2, or 4 if the respective DV showed a susceptible reaction to a blast isolate (i.e., the code 1 was assigned if the first DV in the subunit was susceptible; 2 was assigned if the second DV in the subunit was susceptible; and 4 was assigned if the third DV in the subunit was susceptible). Blast races were designated by the combined sum of the codes representing the reactions of the three DVs in each subunit. Isolates classified this way were designated as "reaction type" within each DV group and as "races" using the set of all five reaction types.

Classification and diversity of blast isolates. Cluster analysis was performed using Ward's hierarchical method (Ward 1963) based on the data on infection scores of the 25 DVs and LTH by blast isolates, using JMP 11.2 for Windows software (2014; SAS Institute Inc., Cary, NC). Relationships of blast isolates between the cluster groups, as well as the ecosystems and geographical distributions, were evaluated.

The diversity of the pathogens was calculated using Simpson's diversity index (Simpson 1949). The index values vary from 0 to 1 , where 0 represents no diversity and 1 represents maximum diversity.

\section{Results}

Collection and classification of isolates. A total of 99 isolates were collected from four geographical regions in Kenya: Kirinyaga County (18 isolates)/Embu County (14 isolates), Kisumu County

Table 1. Reaction types of Pyricularia oryzae isolates to 25 rice differential varieties (DVs) for resistance genes and to variety Lijiangxintuanheigu (LTH) (susceptible control)

\begin{tabular}{|c|c|c|c|c|c|c|c|c|c|c|c|c|c|}
\hline \multirow[b]{3}{*}{ "U" DV group reaction type } & \multirow[b]{3}{*}{ Pish } & & & & & & & & & \multicolumn{4}{|c|}{ No. of blast isolates (\%) } \\
\hline & & \multicolumn{7}{|c|}{ Reaction to resistance gene in differential variety } & & \multicolumn{3}{|c|}{ Cluster group } & \multirow[b]{2}{*}{ Total $(n=99$} \\
\hline & & $P i b$ & Pit & LHT & Pia & & & & & Ia $(n=24)$ & $\mathrm{Ib}(n=62)$ & II $(n=13)$ & \\
\hline U01 & $\mathrm{a}$ & $\mathrm{a}$ & $\mathrm{a}$ & $\mathrm{v}$ & a & - & - & - & - & 23 & 2 & & $25(25.3)$ \\
\hline U43 & a & a & $\mathrm{v}$ & $\mathrm{v}$ & $\mathrm{v}$ & - & - & - & - & & 9 & 10 & $19(19.2)$ \\
\hline U63 & a & $\mathrm{v}$ & $\mathrm{v}$ & $\mathrm{v}$ & $\mathrm{v}$ & - & - & - & - & & 27 & 3 & $30(30.3)$ \\
\hline Sum & - & - & - & - & - & - & - & - & - & 23 & 38 & 13 & $74(74.7)$ \\
\hline $\begin{array}{l}\text { Others (U00, U03, U20, U21, } \\
\text { U23, U40, U41, U60) }\end{array}$ & - & - & - & - & - & - & - & - & - & 1 & 24 & 0 & $25(25.3)$ \\
\hline $\begin{array}{l}\text { Simpson's index of diversity } \\
\text { (1-D) }\end{array}$ & - & - & - & - & - & - & - & - & - & 0.08 & 0.77 & 0.36 & 0.80 \\
\hline “i” DV group reaction type & $P i i$ & $P i 3$ & $P i 5(\mathrm{t})$ & - & - & - & - & - & - & & & & \\
\hline i0 & a & a & $\mathrm{a}$ & - & - & - & - & - & - & 3 & 11 & & $14(14.1)$ \\
\hline i3 & $\mathrm{v}$ & $\mathrm{v}$ & $\mathrm{a}$ & - & - & - & - & - & - & 8 & 3 & 2 & $13(13.1)$ \\
\hline i7 & $\mathrm{v}$ & $\mathrm{v}$ & $\mathrm{v}$ & - & - & - & - & - & - & 10 & 34 & 4 & $48(48.5)$ \\
\hline Sum & - & - & - & - & - & - & - & - & - & 21 & 48 & 6 & $75(75.8)$ \\
\hline Others (i1, i2, i4, i5, i6) & - & - & - & - & - & - & - & - & - & 3 & 14 & 7 & $24(24.2)$ \\
\hline $\begin{array}{l}\text { Simpson's index of diversity } \\
\text { (1-D) }\end{array}$ & - & - & - & - & - & - & - & - & - & 0.69 & 0.67 & 0.66 & 0.71 \\
\hline "k" DV group reaction type & $P i k-s$ & - & - & Pik-m & Pil & Pih-h & Pik & Pik-p & $\operatorname{Pik} 7(\mathrm{t})$ & & & & \\
\hline k177 & $\mathrm{v}$ & - & - & $\mathrm{v}$ & $\mathrm{v}$ & $\mathrm{v}$ & $\mathrm{v}$ & $\mathrm{V}$ & $\mathrm{v}$ & 20 & 29 & & $49(49.5)$ \\
\hline $\begin{array}{l}\text { Others (k000, k002, k0003, } \\
\text { 1037, k055, k076, k077, } \\
\text { k100, k102, k104, k106, } \\
\text { k107, k120, k134, k136, } \\
\text { k137, k152, k153, k157, } \\
\text { k175) }\end{array}$ & - & - & - & - & - & - & - & - & - & 4 & 33 & 13 & $50(50.5)$ \\
\hline $\begin{array}{l}\text { Simpson's index of diversity } \\
\text { (1-D) }\end{array}$ & - & - & - & - & - & - & - & - & - & 0.30 & 0.76 & 0.77 & 0.73 \\
\hline “z” DV group reaction type & $P i 9(\mathrm{t})$ & - & - & $P i z$ & $P i z-5$ & $P i z-t$ & - & - & - & & & & \\
\hline z00 & a & - & - & $\mathrm{a}$ & a & $\mathrm{a}$ & - & - & - & 24 & 47 & & $71(71.7)$ \\
\hline z01 & $\mathrm{a}$ & - & - & $\mathrm{v}$ & $\mathrm{a}$ & $\mathrm{a}$ & - & - & - & & 7 & 13 & $20(20.2)$ \\
\hline Sum & - & - & - & - & - & - & - & - & - & 24 & 54 & 13 & $91(91.9)$ \\
\hline Others (z02, z04, z10) & - & - & - & - & - & - & - & - & - & 0 & 8 & 0 & $8(8.1)$ \\
\hline $\begin{array}{l}\text { Simpson's index of diversity } \\
\text { (1-D) }\end{array}$ & - & - & - & - & - & - & - & - & - & 0.00 & 0.44 & 0.00 & 0.46 \\
\hline "ta" DV group reaction type & $\begin{array}{l}\text { Pita- } \\
2(\mathrm{Pi})\end{array}$ & $\begin{array}{l}\text { Pita-2 } \\
(\mathrm{Re})\end{array}$ & $\operatorname{Pi12}(\mathrm{t})$ & $\begin{array}{l}\text { Pita } \\
(\mathrm{K} 1)\end{array}$ & $\begin{array}{l}\text { Pita } \\
\text { (CP1) }\end{array}$ & - & $\operatorname{Pi19}(\mathrm{t})$ & $P i 20(\mathrm{t})$ & - & & & & \\
\hline $\mathrm{ta} 003$ & a & a & $\mathrm{a}$ & a & a & - & $\mathrm{v}$ & $\mathrm{v}$ & - & & 23 & & $23(23.2)$ \\
\hline ta403 & $\mathrm{a}$ & a & $\mathrm{v}$ & a & a & - & $\mathrm{v}$ & $\mathrm{v}$ & - & & 10 & & $10(10.1)$ \\
\hline ta431 & $\mathrm{a}$ & $\mathrm{a}$ & $\mathrm{v}$ & $\mathrm{v}$ & $\mathrm{v}$ & - & $\mathrm{v}$ & $\mathrm{a}$ & - & & & 11 & $11(11.1)$ \\
\hline Sum & - & - & - & - & - & - & - & - & - & 0 & 33 & 11 & 44 (44.4) \\
\hline $\begin{array}{l}\text { Others }(\mathrm{ta} 000, \operatorname{ta} 001, \operatorname{ta} 002, \\
\text { ta013, } \mathrm{ra} 031, \operatorname{ta} 033, \operatorname{ta} 121, \\
\text { ta131, ta301, ta311, ta321, } \\
\text { ta331, ta400, ta402, ta421, } \\
\text { ta433, ta723) }\end{array}$ & - & - & - & - & - & - & - & - & - & 24 & 29 & 2 & 55 (55.6) \\
\hline $\begin{array}{l}\text { Simpson's index of diversity } \\
\text { (1-D) }\end{array}$ & - & - & - & - & - & - & - & - & - & 0.75 & 0.81 & 0.26 & 0.89 \\
\hline
\end{tabular}

\footnotetext{
${ }^{\mathrm{a}} \mathrm{a}=$ avirulent and $\mathrm{v}=$ virulent.
} 
(26 isolates), Tana River County (11 isolates), and Mombasa County (12 isolates), plus two isolates from an unknown region. These isolates showed different frequencies of virulence against the $25 \mathrm{DVs}$ and LTH (Fig. 1). High frequencies of virulence $(>50 \%)$ were seen in the 14 DVs for Pib, Pia, Pii, Pi3, Pi5(t), Pik-s, Pik-m, Pil, Pik-h, $P i k$, Pik-p, Pi7(t) Pi19(t), and Pi2O(t); intermediate frequencies of virulence (10 to 50\%) were seen in the seven DVs for Pit, Piz, Pita-2 (two lines), Pi12(t), and Pita (two lines); and low frequencies of virulence $(<10 \%)$ were seen in the four DVs for Pish, Pi9(t), Piz-5, and Piz-t. The control variety LTH was susceptible to $96.0 \%$ of the isolates and showed resistance to the others. The results indicate that the reactions to resistance genes in the DVs varied among the blast isolates tested.

Based on their patterns of reaction to the $25 \mathrm{DVs}$ and $\mathrm{LTH}$, the isolates were classified into two cluster groups (clusters I and II) and then divided again into three subgroups (Ia, Ib, and II) (Supplementary Fig. S1). These cluster groups showed different frequencies of isolates virulent for each DV (Fig. 1). Cluster groups Ia and Ib included 24 and 62 of the isolates, respectively, and showed minor differences in the frequencies of virulence for several DVs. Cluster group Ia differed from group $\mathrm{Ib}$ in that there were several isolates avirulent to LTH in group $\mathrm{Ib}$ and the frequencies of isolates virulent for DVs for Pib, Pit, Pia, and Pi2O(t) were remarkably higher in group Ib than Ia, whereas the frequencies of isolates virulent for DVs for Pi3, Pita-2 (two lines), and Pita (two lines) were higher in group Ia than in group Ib. Cluster group II included only 13 isolates. The low virulent isolates for DVs for Pii, Pik-m, Pil, Pik-h, Pik, Pik$p$, and $P i 7(\mathrm{t})$ and highly virulent isolates for DVs for Pib, Pit, Piz, $P i 12(\mathrm{t})$, and Pita (two lines) were remarkably different between subgroups Ia and $\mathrm{Ib}$.

Distributions of cluster groups. Distributions of the cluster groups differed among the four geographical regions in Kenya (Fig. 2). Subgroup Ib was found in all four regions (Kisumu County, Kirinyaga County/Embu County, Tana River County, and Mombasa County), with frequencies of $43.8,90.5,9.1$, and $75.0 \%$, respectively. Subgroup Ia was found only in two regions (Kisumu County and Kirinyaga County/Embu County), with frequencies of 56.3 and $9.5 \%$, respectively. Group II was also distributed in only two regions (Mombasa County and Tana River County), with frequencies of 28.0 and $90.9 \%$, respectively.

Reaction types in groups of DVs and diversity. Several predominant reaction types of isolates were found in each group of DVs, and the diversities of isolates differed among the three cluster groups and four regions for each of the five groups of DVs (Table 1; Supplementary Table S2).

A total of 11 reaction types were found in the group U DVs: U00, U01, U03, U20, U21, U23, U40, U43, U41, U60, and U63. Reaction types U01 (virulent for LTH), U43 (virulent for LTH and the DVs for Pit and Pia), and U63 (virulent for LTH, Pib, Pit, and Pia) had 25, 19 , and 30 blast isolates, respectively. U01, U43, and U63 were

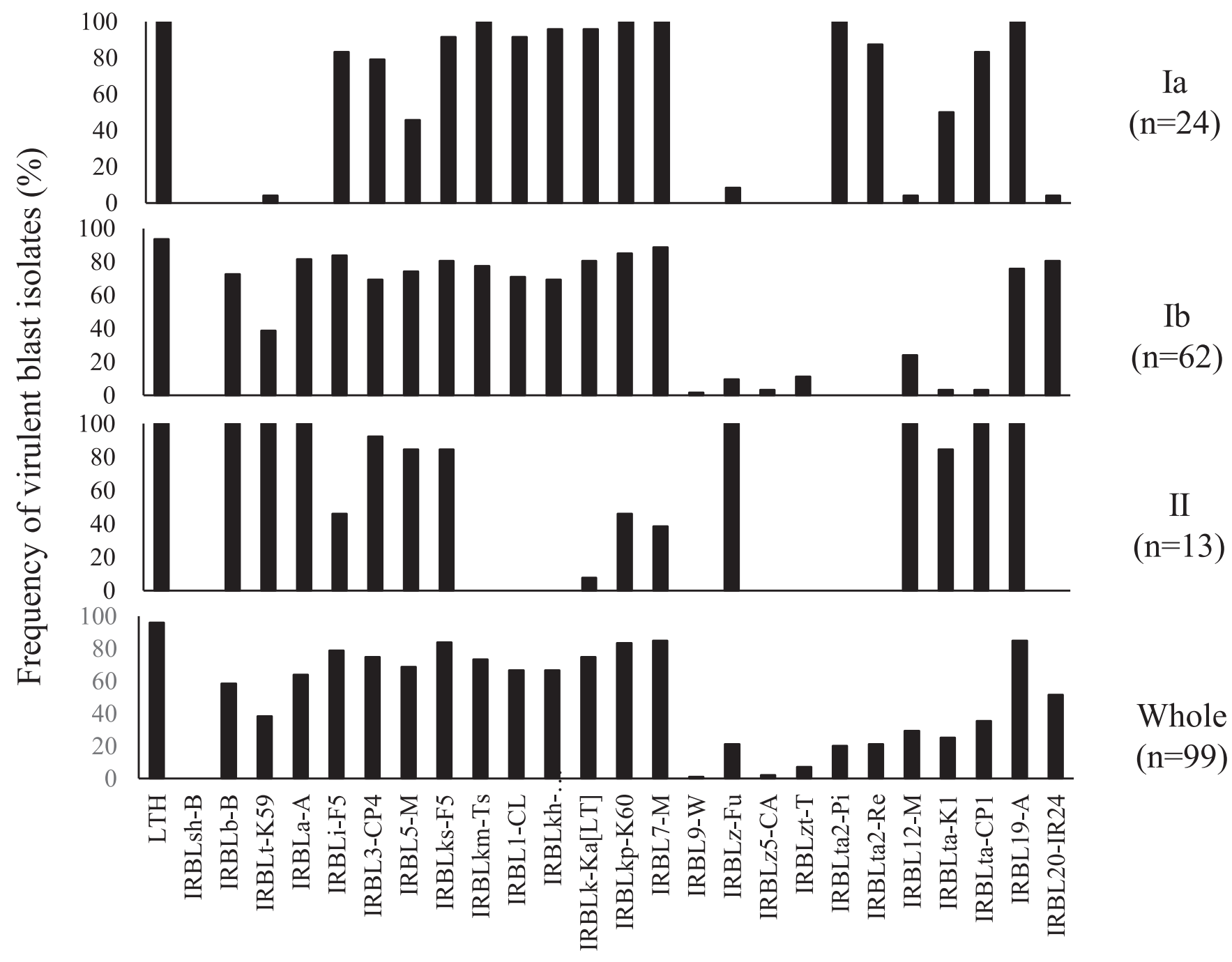

\section{Differential variety and susceptible control}

Fig. 1. Frequencies of blast isolates virulent to differential varieties and susceptible control (Lijiangxintuanheigu [LTH]) in each cluster group. 
shown to be the predominant type in cluster groups Ia, Ib, and II, respectively. The diversity indexes for isolates in cluster groups Ia, Ib, and II and for all isolates as a whole were $0.08,0.77,0.36$, and 0.80 , respectively. The reaction type U01 was distributed mainly in Kirinyaga County/Embu County and U43 and U63 were distributed in Tana River County and Kisumu County, respectively. The diversity index in Tana River County was 0.17 , and it was quite low compared with the other counties. A total of eight reaction types (i0, i1, i2, i3, i4, i5, i6, and i7) were found in group i DVs. The dominant reaction types in this group were i0 with 14 isolates [avirulent for all three DVs in the i group: Pii, Pi3, and Pi5(t)], i3 with 13 isolates [virulent for DVs for Pii and Pi3], and i7 with 48 isolates [virulent for all three of these DVs]. Reaction type i0 was found mainly in cluster group Ib, i3 was found in group Ia, and i7 was found in Ia and Ib. The diversity indexes for isolates in cluster groups Ia, Ib, and II and as a whole were $0.69,0.67,0.66$, and 0.71 , respectively. The diversity index in Kisumu County was 0.26 , which had quite low values compared with the other counties. The low diversity in Kisumu County was induced according to an occurrence of the highly virulent reaction type i7.

In the group $\mathrm{k}$ DVs, a total of 21 reaction types were found $(\mathrm{k} 000$, k002, k003, k037, k055, k076, k077, k100, k102, k104, k106, k107, $\mathrm{k} 120, \mathrm{k} 134, \mathrm{k} 136, \mathrm{k} 137, \mathrm{k} 152, \mathrm{k} 153, \mathrm{k} 157, \mathrm{k} 175$, and k177), and the highly virulent reaction type $\mathrm{k} 177$ was predominant. Reaction type k177 (virulent for all DVs of group k) was found for 20 isolates in cluster group Ia and 29 in group $\mathrm{Ib}$, but there were no isolates in group II. The diversity indexes for isolates in cluster groups Ia, Ib, and II and for all isolates as a whole were 0.30, 0.76, 0.77, and 0.73 , respectively. The reaction type $\mathrm{k} 177$ was predominant in Kisumu County and Kirinyaga County/Embu County, with 26 and
20 isolates, respectively, and the diversity indexes were 0.58 in Kisumu County and 0.59 in Kirinyaga County/Embu County. These were lower than those of the other regions.

In the group z DVs, a total of six reaction types were found (z00, $\mathrm{z} 01, \mathrm{z} 02, \mathrm{z} 04, \mathrm{z} 06$, and z10), and two low virulent types (z00 and z01) were predominant. Reaction type z0 (avirulent for all DVs) included 24 isolates in cluster group Ia and 47 in group Ib. Reaction type z01 (virulent only for the DV for Piz) was found for seven isolates in cluster group Ia and 13 in group II. The diversity indexes for isolates in cluster groups Ia, Ib, and II and for all isolates as a whole were $0.00,0.44,0.00$, and 0.46 , respectively, and these were the lowest values among those of the five DV groups. The diversity indexes among the four counties were quite low (from 0.00 to 0.39 ), except for Mombasa (0.61) where several reaction types were found. These results suggest that there is little differentiation of blast isolates for DV group z compared with the other DV groups, but some variation was found in cluster group Ib and Mombasa County.

In the ta DV group, a total of 20 reaction types were found (ta000, ta001, ta002, ta003, ta013, ta031, ta033, ta121, ta131, ta301, ta311, ta321, ta331, ta400, ta402, ta403, ta421, ta431, ta433, and ta723), and three types among them were predominant: ta003 [virulent for DVs for Pi19(t) and Pi2O(t)], ta403 [virulent for DVs for Pi12(t), $P i 19(\mathrm{t})$, and Pi2O(t)], and ta431 [virulent for DVs for Pi12(t), Pita (two lines), and $P i 19(\mathrm{t})]$. The diversity indexes for isolates in cluster groups Ia, Ib, and II and for all isolates as a whole were $0.75,0.81$, 0.26 , and 0.89 , respectively. The diversity indexes varied from 0.31 to 0.86 among the four regions, and Tana River County showed the lowest value (0.31). The reaction type ta431 was dominant in Tana River County. These results indicate that the low diversities

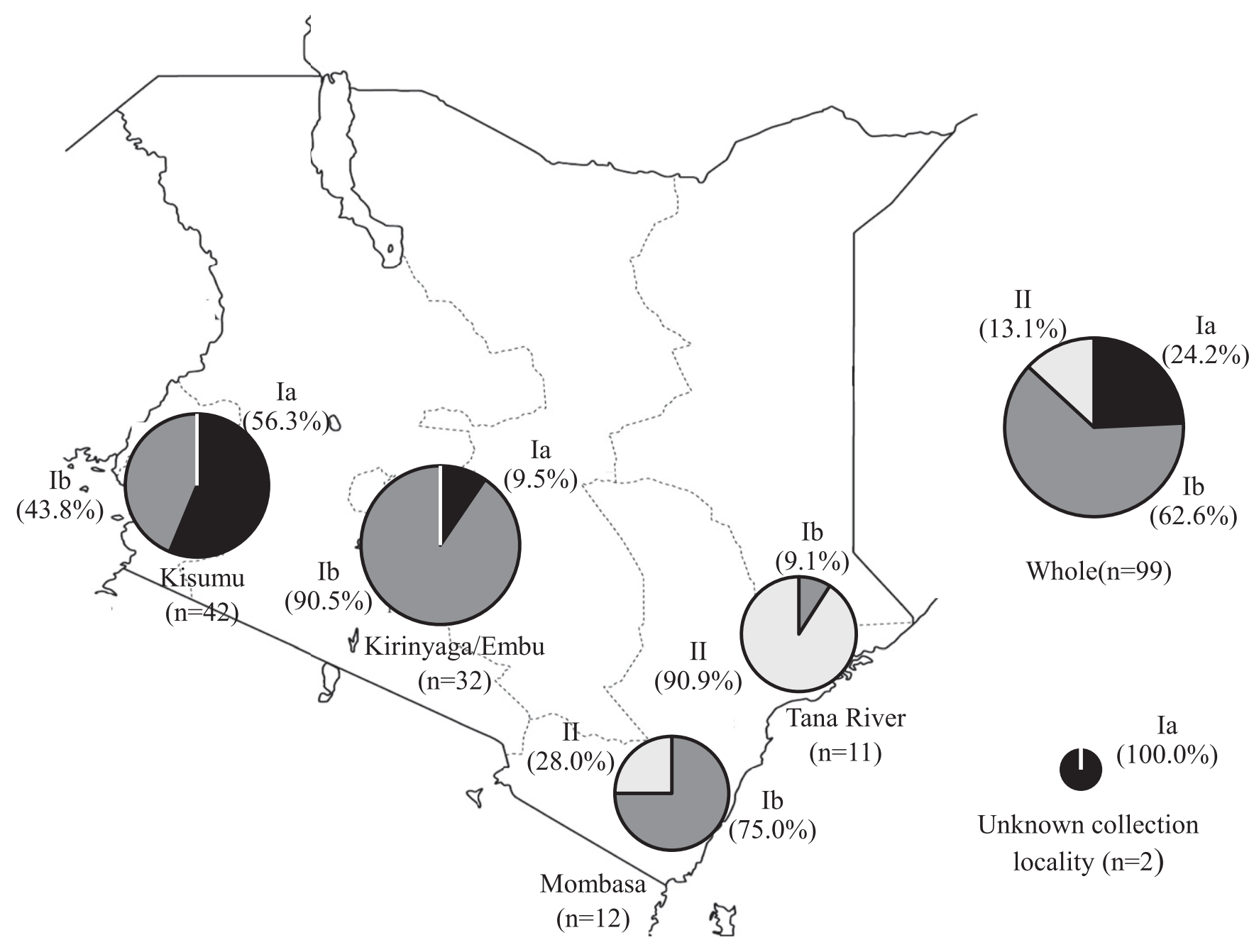

Fig. 2. Distributions of blast isolates classified into four cluster groups in Kenya. 
in cluster group II and Tana River County were induced by the occurrence of the highly virulent and predominant reaction type ta431.

Thus, three cluster groups and four regions of Kenya were characterized by the presence of predominant reaction types. The diversity indexes were also decreased according to the occurrence of predominant reaction types among the cluster groups and four regions.

Blast races. A total of 62 races were found among the 99 blast isolates: 14 races in cluster group Ia, 38 in Ib, and 10 in II (Table 2). A total of 19 blast isolates were categorized into race U63-i7-k177-z00ta003, but the other races included only from one to six isolates each. The isolates in race U63-i7-k177-z00-ta003 were collected in Kisumu County from 2011 to 2012 and infected the nine DVs for Pia, Pii, Pi3, Pik, Pik-m, Pik-h, Pil, Pi19(t), and Pi20(t), as well as Basmati 370 and a hybrid progeny derived from a cross between Habataki and Basmati 370.

\section{Discussion}

A total of 99 blast isolates from four regions in Kenya showed different frequencies of virulence against 25 DVs and LTH. The frequency of virulent isolates was high $(>50 \%)$ with respect to the 14 DVs for Pib, Pia, Pii, Pi3, Pi5(t), Pik-s, Pik-m, Pil, Pik-h, Pik, Pik-p, Pi7(t), Pi19(t), and Pi20(t); intermediate (10 to 50\%) with respect to the seven DVs for Pit, Piz, Pita-2 (two lines), Pi12(t), and Pita (two lines); and low $(<10 \%)$ with respect to the four DVs for Pish, Pi9(t), Piz-5, and Piz-t (Fig. 1). Nyongesa et al. (2016) reported that DVs for Pib, Pit, Pish, Pi5(t), Pik-s, Pik-m, Pil, Pik-h, Pik, Pik-p, $P i z, P i z-5, P i z-t, P i 12(\mathrm{t})$, and Pita-2 were relatively resistant to the population of blast fungus at three locations, based on their field observations: Mwea in Kirinyaga County and West Kano and Gamba in
Kisumu County/Busia County. Our results for the whole collection of 99 isolates from Kenya corresponded mostly with those of Nyongesa et al. (2016), but the reactions to DVs for Pib, Pi5(t), Pik-s, Pik-m, Pil, Pik-h, Pik, and Pik-p were different. These results indicate the instability or limitations of field evaluations based on natural infection and the importance of pathological analysis based on inoculation tests.

Based on the reaction patterns to DVs and LTH, these blast isolates were classified into three cluster groups (Ia, Ib, and II), and the distributions of blast cluster groups differed among the four regions (Fig. 2). Cluster group Ia was found in two regions (Kisumu County and Kirinyaga County/Embu County), and it was the main group in Kisumu County. Cluster group Ib was found in each of the four regions, and II was distributed only in Tana River County (where it was the main group) and Mombasa County. These results indicate that $\mathrm{Ib}$ was distributed as a common or basic group of the blast population in Kenya, and the distributions of blast populations were completely different between the two regions of Tana River County and Mombasa County and the two regions of Kirinyaga County/ Embu County and Kisumu County. Thus, the distributions of cluster groups changed among the four regions in Kenya.

The three cluster groups and four geographical regions were characterized by the predominant reaction types of isolates (Table 1). The presence or absence of reaction types i7 and k177 as well as z00 and z001 were the main differences between cluster groups I and II. The presence or absence of i0, ta003 and ta403, and U01 and U63 were the main differences between $\mathrm{Ia}$ and $\mathrm{Ib}$. Predominant reaction types U63, i7, k177, z00, and ta003 were distributed in Kisumu County; U01, i3, k177, and z00 were in Kirinyaga County/Embu County;

Table 2. Pyricularia oryzae races identified in Kenya

\begin{tabular}{|c|c|c|c|c|c|}
\hline $\begin{array}{l}\text { Cluster } \\
\text { group }\end{array}$ & Race designation & $\begin{array}{c}\begin{array}{c}\text { No. of } \\
\text { isolates }\end{array} \\
\end{array}$ & Cluster group & Race designation & $\begin{array}{c}\text { No. of } \\
\text { isolates }\end{array}$ \\
\hline \multirow[t]{16}{*}{ Ia } & U01-il-k177-z00-ta301 & 1 & $\mathrm{Ib}$ & U23-i5-k153-z00-ta001 & 1 \\
\hline & U01-i0-k136-z00-ta311 & 1 & & U23-i7-k177-z00-ta402 & 1 \\
\hline & U01-i0-k177-z00-ta321 & 2 & & U40-i4-k100-z00-ta000 & 1 \\
\hline & U01-i2-k177-z00-ta321 & 1 & & U41-i0-k000-z01-ta400 & 1 \\
\hline & U01-i3-k157-z01-tal31 & 1 & & U41-i6-k177-z04-ta003 & 1 \\
\hline & U01-i3-k177-z00-ta301 & 1 & & U43-i0-k077-z04-ta002 & 1 \\
\hline & U01-i3-k177-z00-ta321 & 3 & & U43-i0-k107-z00-ta033 & 1 \\
\hline & U01-i3-k177-z00-ta331 & 3 & & U43-i0-k177-z00-ta002 & 1 \\
\hline & U01-i5-k177-z01-tal21 & 1 & & U43-i0-k177-z04-ta002 & 1 \\
\hline & U01-i7-k077-z00-ta301 & 1 & & U43-i6-k077-z04-ta002 & 2 \\
\hline & U01-i7-k157-z00-ra031 & 1 & & U43-i7-k000-z00-ta002 & 1 \\
\hline & U01-i7-k177-z00-ta321 & 1 & & U43 -i7-k107-z00-ta033 & 2 \\
\hline & U01-i7-k177-z00-ta331 & 6 & & U60-i0-k120-z01-ta000 & 1 \\
\hline & U41-i7-k177-z00-ta723 & 1 & & U63-i7-k106-z00-ta403 & 1 \\
\hline & Sum & 24 & & U63-i7-k107-z00-ta403 & 3 \\
\hline & Simpson's index of diversity (1-D) & 0.88 & & U63-i7-k107-zl0-ta403 & 1 \\
\hline \multirow[t]{19}{*}{$\mathrm{Ib}$} & U00-i0-k077-z00-ta402 & 1 & & U63-i7-k134-z00-ta403 & 2 \\
\hline & U00-i1-k077-z00-ta003 & 1 & & U63-i7-kl36-z00-ta403 & 1 \\
\hline & U00-i4-k000-z01-ta002 & 1 & & U63-i7-k177-z00-ta003 & 19 \\
\hline & U01-i0-k037-z00-ta000 & 1 & & Sum & 62 \\
\hline & U01-i3-k177-z00-ta013 & 1 & & Simpson's index of diversity (1-D) & 0.80 \\
\hline & U01-i4-k076-z00-ta402 & 1 & II & U43-i3-k100-z01-ta431 & 1 \\
\hline & U03-i7-k177-z00-ta033 & 2 & & U43-i4-k106-z01-ta431 & 1 \\
\hline & U03-i7-k177-z04-ta002 & 1 & & U43-i6-k100-z01-ta431 & 2 \\
\hline & U20-i0-k003-z01-ta000 & 1 & & U43-i6-k102-z01-ta421 & 1 \\
\hline & U21-i0-k177-z00-ta001 & 1 & & U43-i6-k106-z01-ta431 & 2 \\
\hline & U21-il-k152-z01-ta000 & 1 & & U43-i6-k107-z01-ta431 & 1 \\
\hline & U21-i3-k137-z00-ta000 & 1 & & U43-i7-k000-z01-ta431 & 1 \\
\hline & U21-i3-k175-z00-ta403 & 1 & & U43-i7-k100-z01-ta431 & 1 \\
\hline & U21-i5-k077-z00-ta000 & 1 & & U63-i7-k104-z01-ta421 & 1 \\
\hline & U21-i5-k157-z00-ta003 & 1 & & U63-i7-k104-z01-ta431 & 2 \\
\hline & U21-i5-k177-z01-ta403 & 1 & & Sum & 13 \\
\hline & U23-il-k055-z02-ta403 & 1 & & Simpson's index of diversity (1-D) & 0.89 \\
\hline & U23-i3-k177-z00-ta003 & 1 & Total & & 99 \\
\hline & U23-i5-k057-z00-ta001 & 1 & $\begin{array}{c}\text { Simpson's index of } \\
\text { diversity (1-D) for whole }\end{array}$ & & 0.92 \\
\hline
\end{tabular}


and U43, z01, and ta431 were in Tana River County. The nondominant type was in Mombasa County. These results indicated that predominant reaction types between cluster groups and geographical regions corresponded, and different reaction types were distributed among the four regions. Particularly, the reaction types of two regions (Kisumu County and Kirinyaga County/Embu County) were completely different from those of Tana River County and Mombasa County.

Low diversity indexes were found in the DV groups $\mathrm{i}, \mathrm{k}$, and $\mathrm{z}$ in Kisumu County; $\mathrm{k}$ and $\mathrm{z}$ in Kirinyaga County/Embu County; and U, $\mathrm{z}$, and ta in Tana River County when comparing those of Mombasa County, except for those of DV group ta in Kisumu County and Kirinyaga County/Embu County. The diversity indexes were decreased according to the occurrences of predominant reaction types among the cluster groups and four regions. Particularly, the lowest diversity indexes for DV groups $\mathrm{i}(0.26)$ and $\mathrm{k}(0.58)$ were found in Kisumu County, and DV groups U (0.17), z (0.00), and ta (0.31) were found in Tana River County. Those in Kirinyaga County/Embu County and Mombasa County showed intermediate or the highest values of diversity indexes among the five DV groups. Kihoro et al. (2013) reported that serious blast infections have been observed in the Mwea, Kirrinyaga/Embu region. These results suggest that there is a high risk for an occurrence of virulent blast races in Kisumu County and Tana River County, followed by Kirinyaga County/Embu County. Mombasa County maintains its high diversity for blast races, and the risk of virulent blast races might be the lowest among the four regions. Kariaga et al. (2016) and Nyongesa et al. (2016) also suggested that different blast populations were distributed in Kenya. Our study demonstrated well the distribution or differentiation of different blast populations among the four regions, and these blast races in Kisumu County, Kirrinyaga/Embu region, and Tana River County, where the cultivations of limited rice cultivars have been continued, were completely different from that of Mombasa County in particular. To our knowledge, this is the first report on the pathogenicities of isolates in Kenya based on inoculation tests using international DVs. Nevertheless, the number of isolates collected and investigated in this study was limited, and more samples need to be collected and the pathogenicities evaluated for a more detailed understanding of the distributions and differentiation of races in Kenya.

The same set of DVs established by Tsunematsu et al. (2000) and Telebanco-Yanoria et al. (2010) was used for the pathogenicity analyses of blast isolates from six regions: Jilin Province in northeastern China (Wang et al. 2013), Western Africa (Odjo et al. 2014), Cambodia (Fukuta et al. 2014), the United States (Wang et al. 2015), Japan (Kawasaki-Tanaka et al. 2016), and Bangladesh (Khan et al. 2016). The frequencies of isolates virulent on DVs for $P i 9(\mathrm{t})$ were constantly low, and those for DVs for Pia, Pii, Pi3, Pi5(t), and Pi2O(t) were commonly high among seven regions including Kenya in this study. However, the frequencies with respect to DVs for other resistance genes differed. These results suggest that $\mathrm{Pi} 9$ is the most stable resistance gene. On the other hand, virulent isolates against the resistance genes Pia, Pii, Pi3, Pi5(t), and Pi2O(t) were distributed widely in these seven regions. The other virulent isolates for DVs for Pish, Pib, Pit, Pik-s, Pik-m, Pil, Pik-h, Pik, Pik-p, Pi7(t), Piz, Piz-5, Piz-t, Pita-2, Pita, Pi12(t), and Pi19(t) changed among these regions. The frequencies of virulent isolates were compared among the seven regions, and these results indicate the tendencies or differences in the variation of isolates among them, and the importance of using the common DV set.

These isolates from the four regions of Kenya, Cambodia, Japan, Bangladesh, and Western Africa were designated as reaction types for each DV group and race, based on the designation system by Hayashi and Fukuta (2009). Fukuta et al. (2014) analyzed the pathogenicities of 122 isolates from Cambodia and found that U43, U61, U63, i0, i6, i7, k000, k100, z00, z04, z06, ta002, ta033, ta403, ta421, and ta431 were the predominant reaction types. Odjo et al. (2014) clarified the pathogenicities of 96 isolates from Western Africa and found 15 reaction types (U23, U43, U63, i0, i7, k100, k106, k177, z00, z03, z04, ta003, ta031, ta403, and ta431). In Japan, Kawasaki-Tanaka et al. (2016) examined 310 isolates and found 11 predominant reaction types (U11, U13, U33, i0, i7, k100, k177, z00, z10, ta001, and ta401). In Bangladesh, Khan et al. (2016) investigated the pathogenicities of 331 isolates and found 26 predominant reaction types (U03, U23, U43, U61, U63, U73, i0, i4, i7, z00, z02, z04, z05, z06, $\mathrm{z} 07, \mathrm{z} 17, \mathrm{k} 000, \mathrm{k} 100, \mathrm{k} 102, \mathrm{k} 177, \mathrm{ta} 003, \mathrm{ta} 023, \mathrm{ta} 402, \mathrm{ta} 403$, ta423, and ta431). In Kenya, 12 reaction types (U01, U43, U63, i0, i3, i7, k177, z00, z01, ta003, ta403, and ta431) were predominant. Three predominant reaction types, i0 [avirulent for $P i i, P i 3$, and $P i 5(\mathrm{t})]$, i7 [virulent for $P i i, P i 3$, and $P i 5(\mathrm{t})$ ], and $\mathrm{z} 00$ [avirulent for Pi9, Piz, $P i z-5$, and $P i z-t]$, were common to all five regions. These results suggest that rice cultivars harboring the three resistance genes Pii, Pi3, and $P i 5(\mathrm{t})$ would be more vulnerable to an occurrence of virulent blast races compared with cultivars harboring the other genes, whereas an outbreak of virulent races would be more difficult in rice cultivars harboring the four resistance genes Pi9, Piz, Piz-5, and Piz-t. These results correspond with the frequencies of virulent isolates for DVs among the five regions. In addition, six predominant reaction types, U43 [virulent for Pit, Pia, and LTH], U63 [virulent for Pit, Pib, LTH, and Pia], k100 [virulent for Pik-s], k177 [virulent for Pik-s, Pik-m, Pil, Pik-h, Pik, Pik-p, and Pi7(t)], ta403 [virulent for $P i 12(\mathrm{t}), P i 19(\mathrm{t})$, and $P i 20(\mathrm{t})$ ], and ta431 [virulent for $P i 12(\mathrm{t})$, Pita(K1), Pita(CP1), and Pi19(t)], were also found in four regions at least. These results indicate that the virulent races for Pit, Pia, Pib, Pik-s, Pik-m, Pil, Pik-h, Pik, Pik-p, Pi7(t) Pi12(t), Pita (K1), Pita (CP1), Pi19(t), and Pi2O(t) differentiated widely among them. Therefore, these resistance genes are highly susceptible to the occurrence of virulent races and pose a high risk in rice breeding programs. Among nine common reaction types, eight types were found in Kenya. Thus, predominant reaction types changed among five regions with several common tendencies.

The outbreak of blast races can be explained by the gene-for-gene theory: every resistance gene in the host corresponds to an avirulence gene in the pathogen (Flor 1971; Silué et al. 1992). This means that the virulence of a blast isolate is determined by the genotypes of the resistance genes in the rice cultivar. The complex situation of blast populations might be related to the rice cultivars that have been cultivated in each region. These clarified the high risks for an outbreak of predominant reaction types in Kisumu County and Tana River County, followed by Kirinyaga County/Embu County, where monoculture has been conducted under widespread irrigation schemes using limited types of rice cultivars such as Basmati 217, Basmati 370, and ITA310. These isolates from Kenya were categorized finally into 62 races (Table 2). Race U63-i7-k177-z00-ta003 [virulent for DVs for Pib, Pit, Pia, Pii, Pi3, Pi5(t), Pik-s, Pik-m, Pil, Pik-h, Pik, Pik-p, $P i 7(\mathrm{t}), P i 19(\mathrm{t})$, and $P i 20(\mathrm{t})]$ included 19 isolates, whereas other races had few isolates with numbers ranging from 1 to 6 . These isolates were collected from monogenic lines as follows: IRBLa-c for Pia, IRBLiF5 for Pii, IRBL3-CP4 for Pi3, IRBLk-Ka for Pik, IRBLkm-Ts for Pik-m, IRBLkh-K3 for $P i k-h$, IRBL1-CL for Pil, IRBL19-A for Pi19(t), and IRBL20-IR24 for Pi2O(t) and cultivar Basmati 370, and a hybrid progeny line derived from a cross between Habataki and Basmati 370, in Kisumu County. The virulence of race corresponded with the resistance genes in these DVs. This means that Basmati 370 and the hybrid progeny line might harbor some of these resistance genes in their genetic backgrounds, based on the gene-forgene theory. Thus, the monoculture using limited types of rice cultivars might induce high risks in Kisumu County, Tana River County, and Kirinyaga County/Embu County in Kenya. On the other hand, there were no predominant reaction types with high virulence and high diversities observed in almost all DV groups in Mombasa County, where small irrigated or rainfed paddy fields comprised the rice-growing areas. Therefore, in order to develop a strategy for a stable system of protection against blast fungus, it is necessary to further understand the relationships between blast fungus and host plants in Kenya, Thus, it will also be necessary to identify the resistance genes present in rice cultivars that are growing in farmers' fields.

As the above results indicate, we were able to demonstrate the usefulness of the set of international differential DVs developed by Tsunematsu et al. (2000) and Telebanco-Yanoria et al. (2010) and the 
new designation system developed by Hayashi and Fukuta (2009) as common tools for the investigation and pathological analysis of blast isolates and for understanding the differentiation of races on a global level. These findings will be further confirmed as data on the pathogenicity of isolates are accumulated from various countries around the world.

\section{Literature Cited}

Bonman, J. M., Vergel de Dios, T. I., and Khin, M. M. 1986. Physiologic specialization of Pyricularia oryzae in the Philippines. Plant Dis. 70:767-769.

Chen, H. I., Chen, B. T., Zhang, D. P., Xie, Y. F., and Zhang, Q. 2001. Pathotypes of Pyricularia grisea in rice fields of central and southern China. Plant Dis. 85: 843-850.

Flor, H. H. 1971. Current status of the gene-for-gene concept. Annu. Rev. Phytopathol. 9:275-296.

Fukuta, Y., Koga, I., Ung, T., Sathya, K., Kawasaki-Tanaka, A., Koide, Y., Kobayashi, N., Obara, M., Yanada, H., and Hayashi, N. 2014. Pathogenicity of rice blast (Pyricularia oryzae Cavara) isolates from Cambodia. JARQ 48: $155-166$.

Gilmour, J. 1973. Octal notation for designating physiologic races of plant pathogens. Nature 242:620.

Hayashi, N., Ando, I., and Imbe, T. 1998. Identification of a new resistance gene to a Chinese blast fungus isolate in the Japanese rice cultivar Aichi Asahi. Phytopathology 88:822-827.

Hayashi, N., and Fukuta, Y. 2009. Proposal for a new international system of differentiating races of blast (Pyricularia oryzae Cavara) by using LTH monogenic lines in rice (Oryza sativa L.). Pages 11-15 in: JIRCAS Working Report No. 63. Japan International Research Center for Agricultural Sciences, Tsukuba, Ibaraki, Japan.

Hayashi, N., Kobayashi, N., Vera Cruz, C. M., and Fukuta, Y. 2009. Protocols for the sampling of diseased specimens and evaluation of blast disease in rice. Pages 17-33 in: JIRCAS Working Report No. 63. Japan International Research Center for Agricultural Sciences, Tsukuba, Ibaraki, Japan.

Kariaga, M. G., Wakhungu, J., and Were, H. K. 2016. Identification of rice blast (Pyricularia oryzae Cav.) races from Kenyan rice growing regions using culture and classical characterization. J. Res. Agric. Anim. Sci. 4:16-24.

Kato, H., Imbe, T., Tsunemasu, H., and Fukuta, Y. 2004. Developing rice blast differential lines and evaluating partial resistance for breeding of durable rice varieties in the tropics. Pages 31-44 in: Blessing From Nature and Science for the Future. IRRI Limited Proc. No. 10. T. Nozoe, Y. Fukuta, and B. Gardy, eds. International Rice Research Institute, Loa Baños, Philippines.

Kawasaki-Tanaka, A., Hayashi, N., Yanagihara, S., and Fukuta, Y. 2016. Diversity and distribution of rice blast (Pyricularia oryzae Cavara) races in Japan. Plant Dis. 100:816-823.

Khan, M. A. I., Ali, M. A., Monsur, A. A., Kawasaki-Tanaka, A., Hayashi, N., Yanagihara, S., Obara, M., Mia, M. A. T., Latif, M. A., and Fukuta, Y. 2016. Diversity and distribution of rice blast (Pyricularia oryzae Cavara) races in Bangladesh. Plant Dis. 100:2025-2033.

Kihoro, J., Bosco, N. J., Murage, H., Ateka, E., and Makihara, D. 2013. Investigating the impact of rice blast disease on the livelihood of the local farmers in greater Mwea region of Kenya. Springerplus 2:308

Kiyosawa, S. 1981. Gene analysis for blast resistance. Oryza 18:196-203.
Kiyosawa, S. 1984. Establishment of differential varieties for pathogenicity test of rice blast fungus. Rice Genet. Newsl. 1:95-96.

Kouko, W. O. 1997. Review of Kenyan Agricultural Research. Vol. 15. Kenya Agricultural Research Institute, Nairobi.

Mekwatanakarn, P., Kositratana, W., and Zeiglaer, R. S. 2000. Pathotype and avirulence gene diversity of Pyricularia grisea in Thailand as determined by rice near-isogenic lines for major resistance genes. Plant Dis. 84:60-70.

Ministry of Agriculture. 2014. National Rice Development Strategy (2009-2018). Ministry of Agriculture, Nairobi, Kenya.

Noda, T., Hayashi, N., Du, P. V., Dinh, H. D., and Van, E. L. 1999. Distribution of pathogenic races of rice blast fungus in Vietnam. Ann. Phytopathol. Soc. Jpn. 65:526-530.

Nyongesa, B. O., Bigirimana, J., Were, B. A., and Murori, R. 2016. Virulence spectrum of populations of Pyricularia oryzae in irrigated rice ecosystems in Kenya. Eur. J. Plant Pathol. 146:911-922.

Odjo, T., Kawasaki-Tanaka, A., Noda, T., Ahohuendo, B. C., Sere, Y., Kumashiro, T., Yanagihara, S., and Fukuta, Y. 2014. Pathogenicity analysis of blast (Pyricularia oryzae Cavara) isolates from West Africa. JARQ 48:403-412.

Onyango, A. O. 2014. Exploring options for improving rice production to reduce hunger and poverty in Kenya. World Environ. 4:172-179.

Silué, D., Notteghem, J. L., and Tharreau, D. 1992. Evidence of gene-for-gene relationship in the Oryza sativa-Magnaporthe grisea pathosystem. Phytopathology 82:577-580.

Simpson, E. H. 1949. Measurement of diversity. Nature 163:688.

Telebanco-Yanoria, M. J., Imbe, T., Kato, H., Tsumematsu, H., Ebron, L. A., Vera Crus, C. M., Kobayashi, N., and Fukuta, Y. 2008. A set of standard differential blast isolates (Magnaporthe grisea (Hebert) Barr.) from the Philippines for rice (Oryza sativa L.) resistance. JARQ 42:23-34.

Telebanco-Yanoria, M. J., Koide, Y., Fukuta, Y., Imbe, T., Kato, H., Tsunematsu, H., and Kobayashi, N. 2010. Development of near-isogenic lines of Japonicatype rice variety Lijiangxintuanheigu as differentials for blast resistance. Breed. Sci. 60:629-638.

Tsunematsu, H., Yanoria, M. J. T., Ebron, L. A., Hayashi, N., Ando, I., Kato, H. Imbe, T., and Khush, G. S. 2000. Development of monogenic lines of rice for blast resistance. Breed. Sci. 50:229-234.

Wang, J., Correll, J. C., and Jia, Y. 2015. Characterization of rice blast resistance genes in rice germplasm with monogenic lines and pathogenicity assays. Crop Prot. 72:132-138.

Wang, J. C., Jia, Y., Wen, J. W., Liu, W. P., Liu, X. M., Li, L., Jiang, Z. Y., Zhang, J. H., Guo, X. L., and Ren, J. P. 2013. Identification of rice blast resistance genes using international monogenic differentials. Crop Prot. 45:109-116.

Ward, J. H. 1963. Hierarchical grouping to optimize an objective function. J. Am. Stat. Assoc. 58:236-244.

Yamada, M., Asaga, H., Takahashi, H., and Koizumi, S. 1979. Pathogenic races of rice blast fungus, Pyricularia oryzae Cavara, isolated from Japan. J. Cent Agric. Exp. Stn. 30:11-29.

Yamada, M., Kiyoasawa, S., Yamaguci, T., Hirano, T., Koabayashi, T., Kushibuchi, K., and Eatanabe, S. 1976. Proposal of a new method of differentiating races of Pyricularia oryzae Cavara in Japan. Amm. Phytopathol. Soc. Jpn. 42:216-219.

Zeigler, R. S., Tohme, J., Nelson, R., Levy, M., and Correa, F. 1994. Linking blast population analysis to resistance breeding: A proposed strategy for durable resistance. Pages 267-292 in: Rice Blast Disease. R. S. Zeigler, S. A. Leong, and P. S. Teng, eds. CABI International, Wallingford, UK. 